\title{
Myeloid cell-specific sirtuin 6 deficiency delays wound healing in mice by modulating inflammation and macrophage phenotypes
}

\author{
Jeung-Hyun Koo ${ }^{1}$, Hyun-Young Jang ${ }^{1}$, Youngyi Lee ${ }^{1}$, Young Jae Moon ${ }^{1}$, Eun Ju Bae², Seok-Kweon Yun ${ }^{3,4}$ and \\ Byung-Hyun Park'
}

\begin{abstract}
We recently reported that myeloid cell-expressed sirtuin 6 (Sirt6) plays a crucial role in M1 macrophage polarization and chemotaxis. Given the prominent role of macrophages during wound repair and macrophage heterogeneity, we hypothesized that a Sirt6 deficiency in myeloid cells would delay skin wound closure by affecting the phenotypes of macrophages in wounds. To address this question, a full-thickness excisional lesion was made in the dorsal skin of myeloid cell-specific Sirt6 knockout (KO) and wild-type mice. Wound closure was delayed in the KO mice, which exhibited less collagen deposition, suppressed angiogenesis, and reduced expression of wound healing-related genes compared to the wild-type mice. Using immunohistochemical, flow cytometric, and gene-expression analyses of macrophage subpopulations from wound tissue, we identified increased infiltration of M1 macrophages with a concomitant decrease in M2 macrophage numbers in the KO mice compared to the wild-type mice. Consistent with the in vivo wound closure defects observed in the $\mathrm{KO}$ mice, keratinocytes and fibroblasts treated with $\mathrm{KO}$ macrophage-derived conditioned medium migrated slower than those treated with wild-type macrophage-derived conditioned medium. An analysis of downstream signaling pathways indicated that impaired Akt signaling underlies the decreased M2 phenotypic switching in KO mice. These results suggest that a macrophage phenotypic switch induced by Sirt6 deficiency contributes to impaired wound healing in mice.
\end{abstract}

\section{Introduction}

Skin wound healing is a highly ordered process comprising several overlapping stages: (i) an inflammatory stage that cleans out debris and bacteria, (ii) a proliferative stage that refills the dermal wound space, and (iii) a longterm remodeling stage that involves the resolution of

\footnotetext{
Correspondence: Seok-Kweon Yun (dermayun@jbnu.ac.kr) or Byung-Hyun Park (bhpark@jbnu.ac.kr)

'Department of Biochemistry and Molecular Biology, Chonbuk National University Medical School, Jeonju, Jeonbuk 54896, Republic of Korea

${ }^{2}$ College of Pharmacy, Woosuk University, Wanju, Jeonbuk 55338, Republic of Korea

Full list of author information is available at the end of the article.

These authors contributed equally: Jeung-Hyun Koo, Hyun-Young Jang,

Youngyi Lee
}

inflammation and reorganization of connective tissue into a scar ${ }^{1}$. Thus, the recruitment of inflammatory cells into the wound site is an initial event in the tissue repair process. Neutrophils form the first line of defense against infection and are a source of proinflammatory cytokines ${ }^{2,3}$. Macrophages also regulate wound healing by producing various growth factors such as transforming growth factor- $\beta$, basic fibroblast growth factor, and platelet-derived growth factor ${ }^{4-6}$. In response to these growth factors, epithelial cells proliferate and migrate to cover the wound, endothelial cells participate in angiogenesis, and fibroblasts contribute to the process of dermal healing ${ }^{7}$. In support of this view, suppressed recruitment of macrophages into wound sites impairs wound healing ${ }^{8,9}$. 
However, due to the release of proinflammatory and cytotoxic mediators, uncontrolled macrophage activity may also be detrimental to tissue repair ${ }^{1}$. Macrophages have been subdivided into two subpopulations based on their distinct gene-expression profiles: classically activated M1 macrophages and alternatively activated M2 macrophages ${ }^{10}$. Interestingly, not only does the number of macrophages infiltrating the wound site change but macrophage phenotypes also shift in the various stages of wound repair. In the inflammatory stage, M1 macrophages initiate an acute inflammatory response, whereas during the proliferative stage, M2 macrophages promote angiogenesis and granulation tissue formation ${ }^{11}$. These findings suggest that proper macrophage polarization is critical to effective wound healing. However, the mechanism that regulates macrophage polarization is unclear.

Sirtuins are a class of NAD-dependent histone deacetylases that consists of seven enzymes (Sirt1 to Sirt7) that differ in their cellular localization. Among the seven sirtuin family members, Sirt6 is localized in the nucleus and is involved in transcriptional silencing, genome stability, and longevity ${ }^{12}$. As a histone deacetylase, Sirt6 deacetylates histone $\mathrm{H} 3$ lysine 9 (H3K9) ${ }^{13}$ and histone H3 lysine $56(\mathrm{H} 3 \mathrm{~K} 56)^{14}$ and represses the transcriptional activities of several transcription factors. As a nonhistone protein deacetylase, Sirt6 deacetylates forkhead box protein $\mathrm{O} 1^{15,16}$, C-terminal binding protein interacting protein ${ }^{17}$, GCN $5^{18}$, pyruvate kinase $\mathrm{M}^{19}$, and GATA binding protein $3^{20}$. Since Sirt6 is a critical determinant of phenotypic switching and the migratory responses of macrophages ${ }^{21}$, we hypothesized that myeloid cell-expressed Sirt6 could play a role in the wound healing process. To test this hypothesis, we constructed myeloid cell-specific Sirt6 knockout $(\mathrm{mS6KO})$ mice and studied the effects of Sirt6 deficiency on cell migration in vitro and inflammation and wound healing in vivo.

\section{Materials and methods}

\section{Animals}

Sirt6 $6^{\text {flox/flox }}$ mice $\left(\mathrm{B} 6 ; 129-\right.$ Sirt $\left.^{\mathrm{tm} 1 \mathrm{Ygu}} / \mathrm{J}\right)$ and LysM-Cre

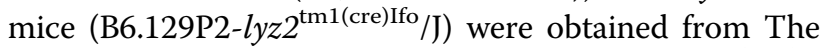
Jackson Laboratory (Bar Harbor, ME, USA). Sirtf flox/flox and homozygous $L y s M$-Cre mice were crossed to obtain $\mathrm{mS6KO}$ mice. To avoid potential variations due to sex and/or genetic background, female mice from the F2 generation [Sirt6 flox/flox; LysM-Cre ${ }^{+}(\mathrm{mS6KO})$ and Sirt6floxflox; $\mathrm{LysM}^{\mathrm{C} C r e^{-}}$(WT)] were used for all experiments. The mice had free access to food and water and were maintained in a room with controlled humidity (50\%) and temperature $\left(22^{\circ} \mathrm{C}\right)$ on a 12 -h light/dark cycle. All animal experiments were performed in accordance with the Guide for the Care and Use of Laboratory Animals published by the US National Institutes of Health (NIH Publication No. 85-23, revised 2011). The study protocol was approved by the Institutional Animal Care and Use Committee of Chonbuk National University (Permit No. CBNU 2015-083).

\section{Wound modeling}

Twelve-week-old WT and KO mice were intraperitoneally anaesthetized with ketamine $(100 \mathrm{mg} / \mathrm{kg}$ ) and xylazine $(5 \mathrm{mg} / \mathrm{kg})$, shaved, and cleaned. An immediatebonding adhesive (Grace Bio-Labs, Bend, OR, USA) was used to fix a splint to the skin. Full-thickness wounds were made in the doughnut region of the splint using 6-mmdiameter punches (Acuderm, Fort Lauderdale, FL, USA). Tegaderm film (3 M Health Care, St. Paul, MN, USA) was placed over the wounds to stabilize the wound site. The percentage of the initial wound that remained open was quantitated at different time points (days $0,3,5,7$, and 14). After the mice were sacrificed, the skin wounds were collected for histopathological analysis and RNA isolation.

\section{Histology}

Tissue was removed and immediately placed in fixative (10\% formalin solution in $0.1 \mathrm{M}$ phosphate-buffered saline $(\mathrm{PBS}))$. Histological sections $(5 \mu \mathrm{m})$ were cut from formalin-fixed, paraffin-embedded tissue blocks. To compare histopathology between lesions, we harvested skin near the center of the wound. The tissue sections were stained with hematoxylin-eosin (H\&E) under standard conditions. Immunohistochemical staining was performed using a DAKO Envision system (DAKO, Carpinteria, CA, USA), which uses dextran polymers conjugated with horseradish peroxidase to avoid contamination with endogenous biotin. After deparaffinization, the tissue sections were treated using a microwave antigen-retrieval procedure with $0.01 \mathrm{M}$ sodium citrate buffer. After blocking endogenous peroxidase activity, the sections were incubated with Protein Block Serum-Free (DAKO) to block nonspecific staining. The sections were then incubated with antibodies against F4/80 and von Willebrand factor (vWF; Millipore, Beverly, MA, USA). Peroxidase activity was detected with 3-amino-9-ethyl carbazole. The number of F4/80-positive macrophages was counted in five microscopic fields (magnification, $100 \times$ ) for each sample in the fields with the highest numbers of F4/80-positive macrophages. The results are expressed as the average number of F4/80-positive macrophages per field. Masson's trichrome staining was performed with a commercial kit from Abcam (ab150686, Cambridge, UK). Double-staining immunofluorescence analysis was performed to determine the types of macrophages in the skin wounds. Frozen sections were incubated with a combination of anti-F4/80 and antiiNOS antibodies (Santa Cruz Biochemicals, Dallas, TX, USA) or anti-F4/80 and anti-MRC1 antibodies (also known as CD206; Abcam) at $4{ }^{\circ} \mathrm{C}$ overnight. 
Myofibroblasts were identified by staining with antibodies to vimentin (Santa Cruz Biochemicals) and $\alpha$-smooth muscle actin ( $\alpha$-SMA; Abcam). After incubation with the corresponding fluorochrome-conjugated secondary antibodies, the sections were mounted and visualized using a LSM510 confocal laser scanning microscope (Carl Zeiss, Oberkochen, Germany).

\section{ELISA analysis}

Tissue levels of TNF- $\alpha$, IL-1 $\beta$, IL-6, IL-4, IL-13, and IL10 were measured using specific ELISA kits (all from eBioscience, San Diego, CA, USA).

\section{Flow cytometric analysis}

Wound cells were isolated by an enzymatic digestion with collagenase V. For flow cytometric analysis, Fc receptors were blocked with mouse SeroBlock FcR (CD16/CD32, eBioscience). The cells were stained with a PerCP- or FITC-conjugated anti-F4/80, a FITC- or PEconjugated anti-Ly6g, an APC-conjugated anti-CD11b, a PE-conjugated anti-Ly6c, or a FITC-conjugated antiMHCII antibody for $30 \mathrm{~min}$ at $4{ }^{\circ} \mathrm{C}$. After washing with FACS buffer (2\% fetal bovine serum (FBS) in PBS) three times, the cells were analyzed using an Accuri flow cytometer (BD Biosciences, San Jose, CA, USA).

\section{Cell culture}

The human keratinocyte cell line $\mathrm{HaCaT}$ was kindly donated by Dr. Jeong HS (Chonnam National University Medical School, Gwangju, Korea). The cells were grown in Dulbecco's modified Eagle's medium (Lonza, Walkersville, MD, USA) supplemented with $10 \%(\mathrm{v} / \mathrm{v})$ FBS and antibiotics $(100 \mathrm{U} / \mathrm{ml}$ penicillin and $100 \mathrm{mg} / \mathrm{ml}$ streptomycin) at $37^{\circ} \mathrm{C}$ in a humidified atmosphere with $5 \% \mathrm{CO}_{2}$. A mouse dermal fibroblast cell line (MDFB) was obtained from iXCells Biotechnologies (San Diego, CA, USA) and grown in fibroblast growth medium (iXCells Biotechnologies). Adenoviruses expressing Sirt6 (AdSirt6) and $\beta$-galactosidase (AdLacZ) were prepared as described previously $^{22}$.

\section{Preparation of conditioned medium and the wound scratch assay}

Bone marrow was isolated from the femurs and tibias of WT and KO mice and cultured in $\alpha$-MEM (Invitrogen, Carlsbad, CA, USA) supplemented with 10\% FBS. Floating cells were defined as bone marrow macrophages (BMMs). To prepare conditioned medium $(\mathrm{CM})$, BMMs $\left(2 \times 10^{6}\right)$ were grown in $\alpha$-MEM supplemented with $10 \%$ FBS. Confluent cells were treated with TNF- $\alpha(10 \mathrm{ng} / \mathrm{ml}), \mathrm{IL}-1 \beta$ $(10 \mathrm{ng} / \mathrm{ml})$, and IL-6 $(10 \mathrm{ng} / \mathrm{ml})$ for $3 \mathrm{~h}$; washed 3 times; and cultured for a further $24 \mathrm{~h}$; then, the supernatants were collected and used. Cell migration was assessed by determining the ability of the cells to move into a cell-free area in a two-dimensional scratch assay. Briefly, $\mathrm{HaCaT}$ cells $\left(2 \times 10^{6}\right.$ cells $)$ or MDFB cells $\left(2 \times 10^{6}\right.$ cells $)$ were grown in a 12 -well plate. When cell confluence reached $90 \%$ or higher, fresh medium containing $10 \mu \mathrm{g} / \mathrm{ml}$ mitomycin $\mathrm{C}$ was added for $2 \mathrm{~h}$. The cells in the center of the well were scratched with a $100-\mu \mathrm{l}$ sterile pipette tip to create a cellfree area. The medium was changed to WT or KO BMMderived $\mathrm{CM}$. The scratched area was photographed using a microscopy system (Carl Zeiss) soon after scratching and 12 and $36 \mathrm{~h}$ later. The scratch area was measured using iSolution DT 36 software (Carl Zeiss).

\section{M2 polarization}

BMMs grown in $\alpha$-MEM were stimulated with IL-4 $(10 \mathrm{ng} / \mathrm{ml}$, Invitrogen) and macrophage colonystimulating factor $(10 \mathrm{ng} / \mathrm{ml}$, Thermo Fisher Scientific, Waltham, MA, USA) for $6 \mathrm{~h}$. To exogenously express Akt in BMMs, cells were transduced with adenoviruses expressing a constitutively active form of Akt (S473D/ T308D, AdAkt). The adenoviruses were a kind gift from Dr. Ahn J.Y. (Sungkyunkwan University, Suwon, Korea) ${ }^{23}$.

\section{Western blotting}

Cells were homogenized in Mammalian Protein Extraction Reagent (Thermo Fisher Scientific). The homogenates $(20 \mu \mathrm{g}$ of total protein) were separated by sodium dodecyl sulphate polyacrylamide gel electrophoresis and transferred to nitrocellulose membranes. The blots were probed with primary antibodies against Sirt6 (Abcam), p-Akt, Akt, p-FoxO1, p-STAT6 (Cell Signaling Technology, Beverly, MA, USA), HSP90, $\alpha$ tubulin, GAPDH (Bioworld, Irving, TX, USA), Arg1 (Santa Cruz Biochemicals), and Ym1 (STEMCELL Technologies, Vancouver, Canada). Immunoreactive bands were detected with a Las-4000 imager (GE Healthcare Life Science, Pittsburgh, PA, USA).

\section{RNA isolation and real-time RT-PCR}

Total RNA was extracted from tissue using Trizol reagent (Invitrogen). RNA was precipitated with isopropanol and dissolved in diethyl pyrocarbonate-treated distilled water. First-strand cDNA was generated with oligo dT-adaptor primers by reverse transcription (TaKaRa). Specific primers were designed using qPrimerDepot (http://mouseprimerdepot.nci.nih.gov, Table S1). The real-time reverse transcription polymerase chain reaction (RT-PCR) reaction systems had a final volume of $10 \mu \mathrm{l}$ and contained $10 \mathrm{ng}$ of reverse-transcribed total RNA, $200 \mathrm{nM}$ forward and reverse primers, and a PCR master mix. RT-PCR was performed in 384-well plates using the ABI Prism 7900HT Sequence Detection System (Applied Biosystems, Foster City, CA, USA). Reverse transcription and PCR were performed using a One-Step RT-PCR kit (Invitrogen). The PCR products were 
separated by electrophoresis on $2 \%$ agarose gels, followed by staining with ethidium bromide.

\section{Statistical analyses}

Data are expressed as the mean \pm standard error of the mean. GraphPad Prism software was used to perform the statistical analyses (GraphPad Prism version 5.2, San Diego, CA, USA). Significant differences between groups were determined using an unpaired Student's $t$ test. A $p$ value less than 0.05 was considered significant.

\section{Results}

\section{Wound healing is impaired in mS6KO mice}

To understand the role of myeloid cell-expressed Sirt6 in excisional wound healing, we generated mS6KO mice by breeding Sirt6 $6^{f l o x / f l o x}$ mice with LysM-Cre mice. By Western blot analysis, the successful deletion of Sirt6 was confirmed in BMMs from mS6KO mice (Fig. 1a). Excision wounds were made in the dorsal skin of female $\mathrm{mS} 6 \mathrm{KO}$ mice and their WT littermates. We observed slower wound healing in the mS6KO mice than in the WT mice, suggesting that the myeloid cell-specific Sirt6 deficiency delayed wound healing (Fig. 1b, c). Histological comparison of the wounds further confirmed delayed wound closure in the $\mathrm{mS6KO}$ mice (Fig. 1d). Trichrome staining of tissue harvested on day 14 after wounding showed reduced collagen content in the granulation tissue of the
mS6KO mice (Fig. 2a). Similarly, the intensity of vWFpositive immunostaining was decreased in $\mathrm{KO}$ tissue, indicating that angiogenesis was also suppressed in the mS6KO mice (Fig. 2a). The mRNA levels of extracellular matrix genes (Col1a1, Col3a1, Timp1, Pdgfra, and Tgfb1) and the angiogenesis gene Vegfa were significantly suppressed in the skin tissue of the mS6KO mice (Fig. 2b). Consistent with these results, the number of myofibroblasts (vimentin ${ }^{+} \alpha-\mathrm{SMA}^{+}$cells) was significantly decreased in the skin wounds of the mS6KO mice (Fig. 2c). Collectively, these data suggest that myeloid cell-specific Sirt6 deficiency delays wound healing through the repression of collagen deposition, epithelial regrowth, and angiogenesis.

\section{Myeloid cell-specific Sirt6 deficiency decreases M2 macrophage infiltration into skin wounds}

We examined the infiltration of macrophages, which are critical inflammatory cells for wound healing, into wound sites by counting F4/80-positive cells. The accumulation of F4/80-immunopositive cells was significantly increased in mS6KO mice compared to WT mice (Fig. 3a, b). Realtime RT-PCR for F4/80 mRNA (Adgre1) expression and flow cytometric analysis for wound-associated macrophages $\left(\mathrm{F} 480^{\mathrm{hi}} \mathrm{Ly} 6 \mathrm{~g}^{\mathrm{lo}} \mathrm{CD} 11 \mathrm{~b}^{\mathrm{hi}}\right.$ ) corroborated the increased accumulation of macrophages in mS6KO mice compared to WT mice (Figs. 3c, d, and S1A).

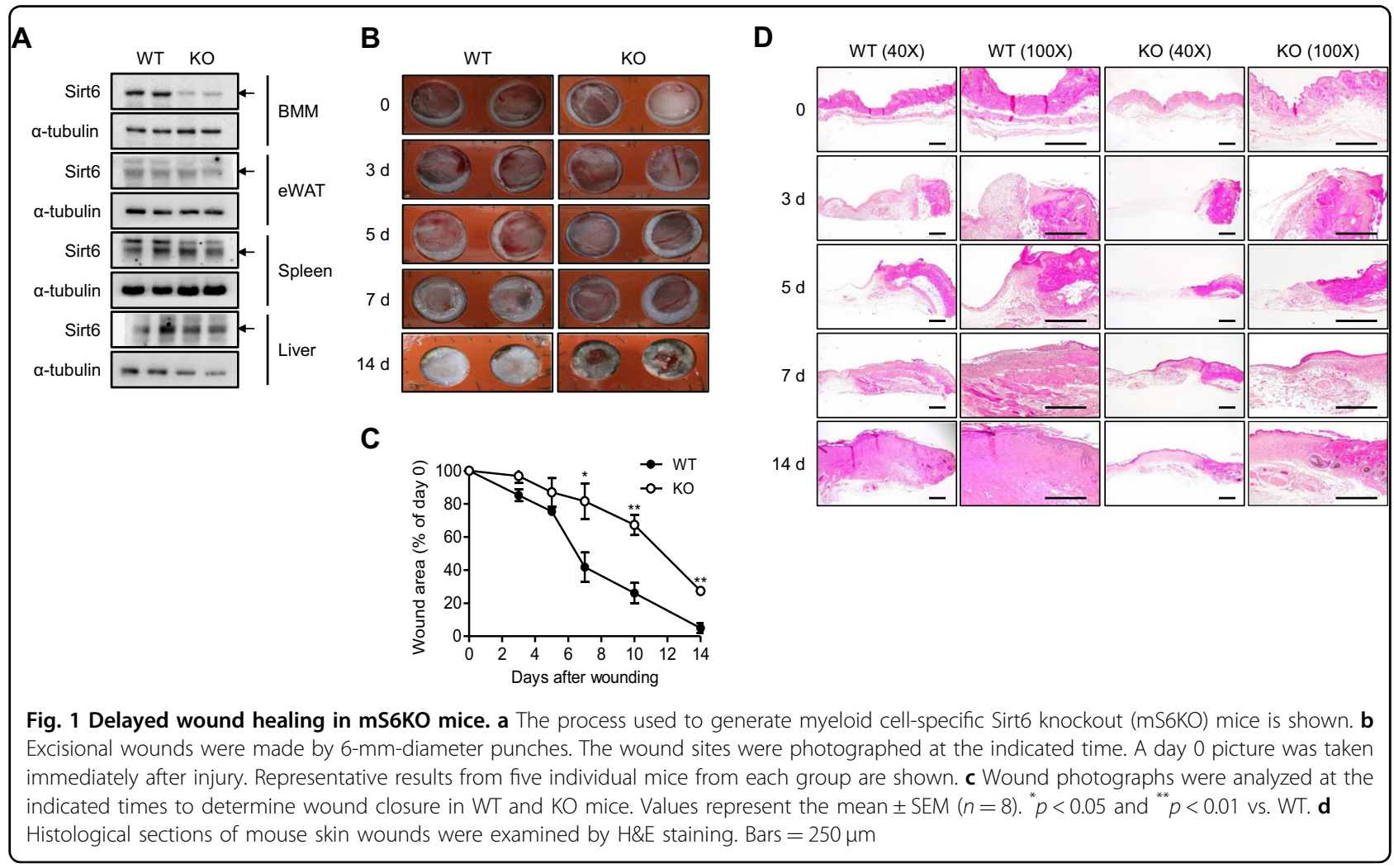



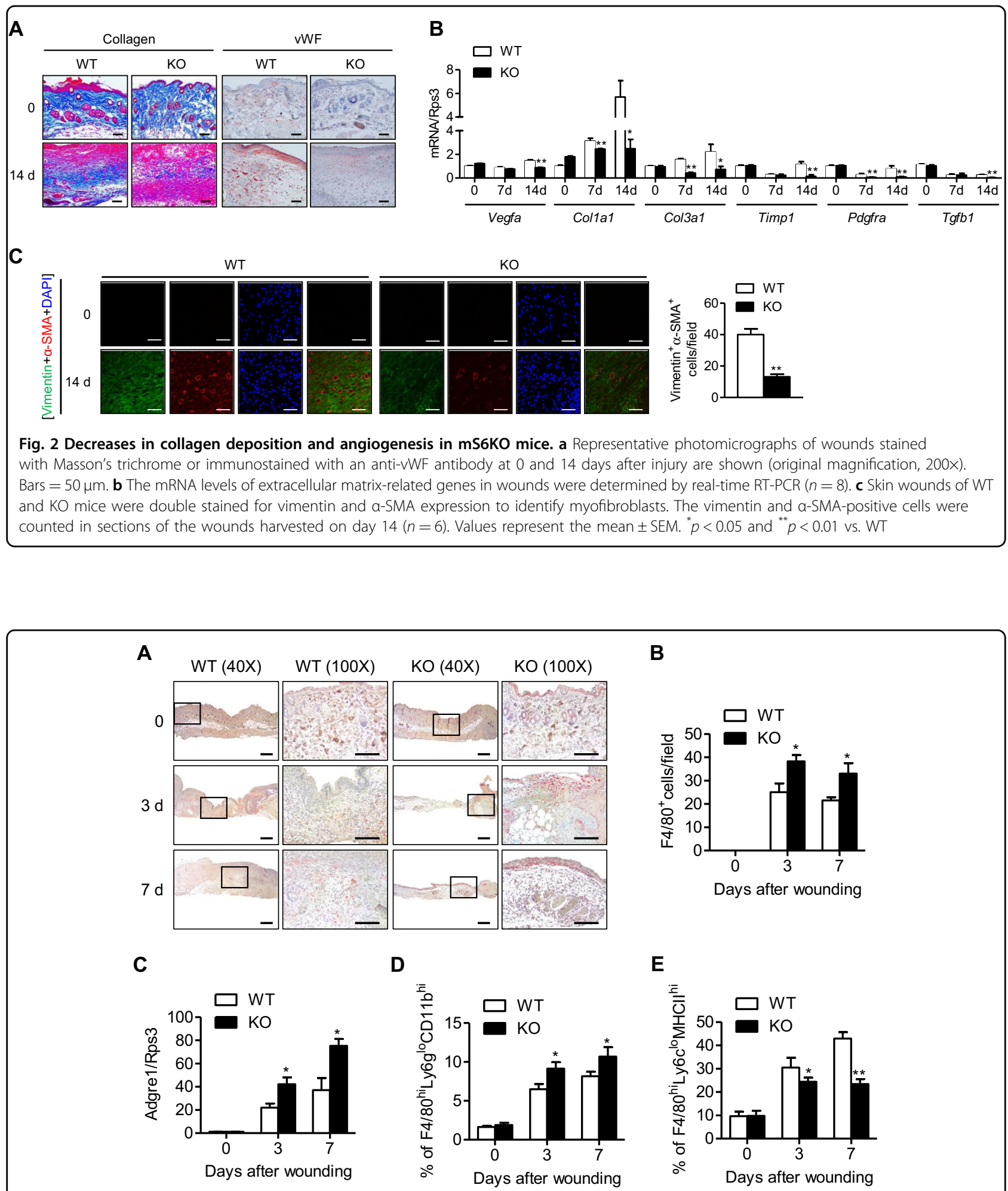

Fig. 3 Increase in macrophage infiltration into wound sites in mS6KO mice. a Immunohistochemistry was used to identify infiltrating macrophages ( $\mathrm{F} 4 / 80^{+}$cells) at 0,3 , and 7 days after injury. Bars $=50 \mu \mathrm{m}$. b F4/80-positive macrophages were counted in wound sections. $\mathbf{c}$ The mRNA level of F4/80 (Adgre1) in the wound site was determined by real-time RT-PCR $(n=8)$. $\mathbf{d}$, e Single-cell suspensions were prepared by enzymatically digesting and gently dissociating skin wounds. After excluding dead cells, the cells were analyzed by flow cytometry to identify

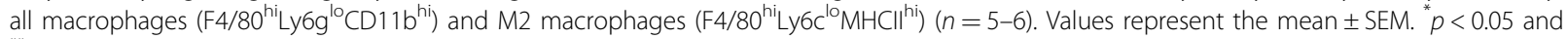
${ }^{* *} p<0.01$ vs. WT 

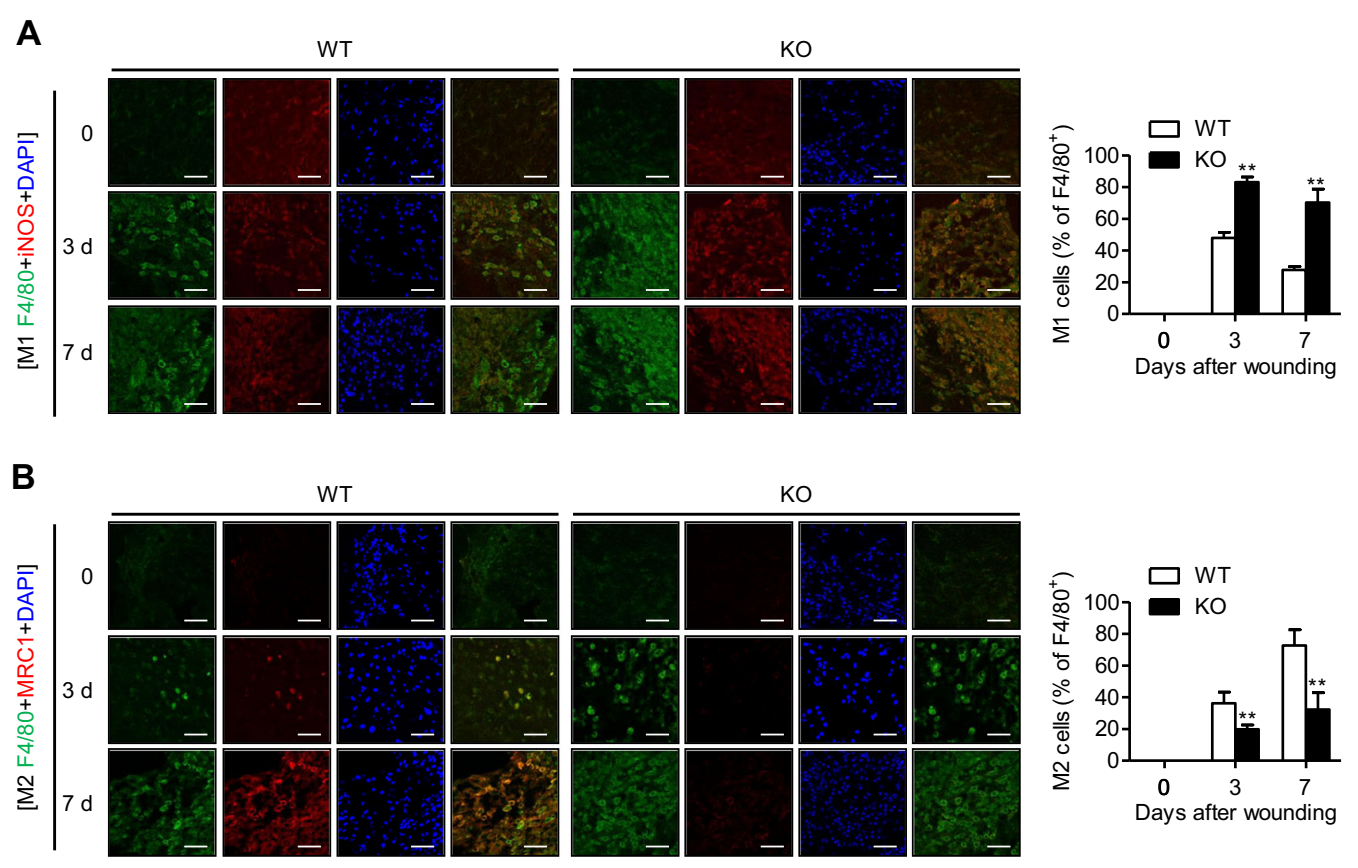

Fig. 4 Decrease in the number of M2 macrophages in wound sites in mS6KO mice. The skin wounds of WT and KO mice were double stained for F4/80 and iNOS to identify M1 macrophages (a) or F4/80 and MRC1 to identify M2 macrophages (b). Digitally merged signals are shown in the right panels. Bars $=50 \mu \mathrm{m}$. Representative results from three independent experiments are shown

Next, we immunostained skin wounds with an M1specific anti-iNOS antibody and M2-specific anti-MRC1 antibody. Myeloid cell-specific Sirt6 deficiency increased the number of $\mathrm{F} 4 / 80^{+}$iNOS $^{+}$macrophages and decreased the number of $\mathrm{F} 4 / 80^{+} \mathrm{MRC1}^{+}$macrophages in the skin wounds of $\mathrm{mS6KO}$ mice compared to those of WT mice (Fig. 4a, b). Flow cytometric analysis also showed a decrease in the accumulation of M2 macrophages (F480 ${ }^{\text {hi }}$ Ly6 $^{\text {lo }} \mathrm{MHCII}^{\text {hi }}$ ) in the skin wounds of mS6KO mice (Figs. 3e and S1B). Consistently, mRNA expression levels for M1 marker genes (Ccl2, Tnfa, Il6, Il1b, Nos2, and Il1b) and M1 cytokines (TNF- $\alpha$, IL-1 $\beta$, and IL-6) were increased, while the levels of M2 marker genes (Clec7a, Arg1, Chil3, Mrc1, and Mgl1) and M2 cytokines (IL-4, IL-13, and IL-10) were decreased in $\mathrm{mS6KO}$ tissue (Fig. $5 \mathrm{a}-\mathrm{d}$ ). These results indicate a decrease in the accumulation of M2-type macrophages in the skin wounds of mS6KO mice.

\section{CM from mS6KO BMMs decreases scratch wound closure}

To examine whether secreted factors from M1 macrophages suppress cell migration during wound closure, we conducted scratch wound assays (Fig. S2). We chose epidermal keratinocytes ( $\mathrm{HaCaT}$ cells) and dermal fibroblasts (MDFB cells) for the cell migration study. CM from $\mathrm{KO}$ BMMs significantly decreased the rate of $\mathrm{HaCaT}$ cell migration compared to CM from WT BMMs (Fig. 6a). Complete gap closure was seen with treatment with $\mathrm{CM}$ from WT BMMs by $36 \mathrm{~h}$, as opposed to treatment with CM from $\mathrm{KO} \mathrm{BMMs}$ resulting in only $58 \%$ closure at the same time. In experiments with MDFB cells, the overall results were similar: the fibroblasts treated with CM from WT BMMs migrated more quickly to fill the scratch area than those treated with CM from KO BMMs (Fig. 6b). Next, we assessed whether exogenous Sirt6 expression in KO BMMs could rescue the migratory defect seen in $\mathrm{HaCaT}$ and MDFB cells. Ectopic overexpression of Sirt6 in KO BMMs rescued cells from the migratory defect (Fig. 6a, b).

\section{Myeloid cell-specific Sirt6 deficiency suppresses M2 polarization through downregulation of the PI3K-Akt pathway}

To explain the decreased number of M2 macrophages in skin wounds in mS6KO mice, we compared M2 polarization in vitro. BMMs isolated from $\mathrm{mS6KO}$ mice expressed markedly lower levels of M2 marker genes (Arg1, Il10, Clec7a, Mrc1, and Chil3) in IL-4-stimulated M2-polarizing conditions than BMMs from WT mice (Fig. 7a). The classic IL-4 signaling pathway involves the activation of phosphoinositide 3-kinase (PI3K)-Akt through the recruitment of IRS $-1 / 2^{24}$. We observed increased levels of p-Akt and the downstream molecule pFoxO1 after IL-4 stimulation in BMMs from WT mice, whereas this increase was not observed in BMMs from mS6KO mice (Figs. 7b and S3A). In addition to activating Akt, IL-4 treatment also leads to the activation of JAK2STAT6 $^{25}$. The phosphorylation levels of STAT6 were 


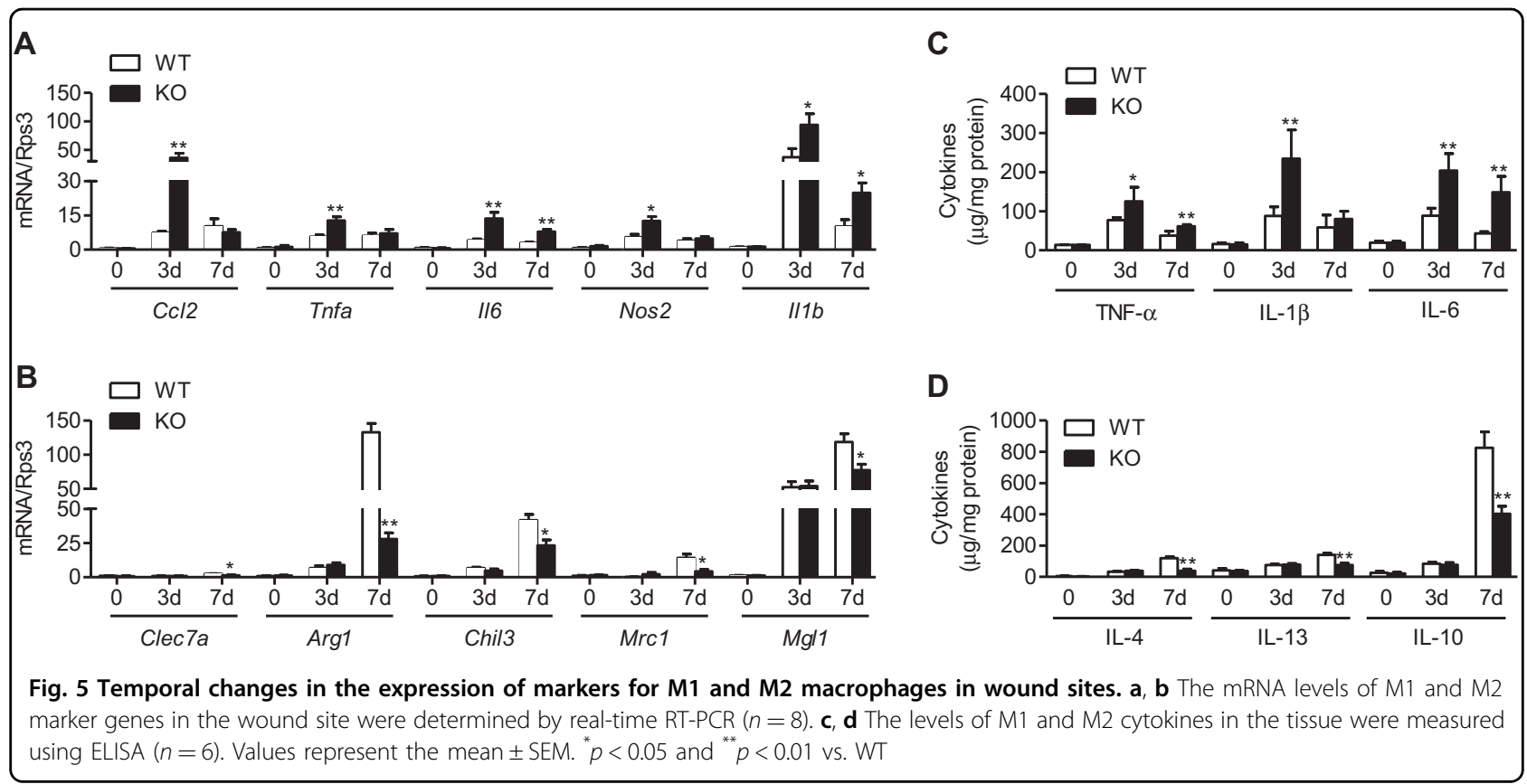

A
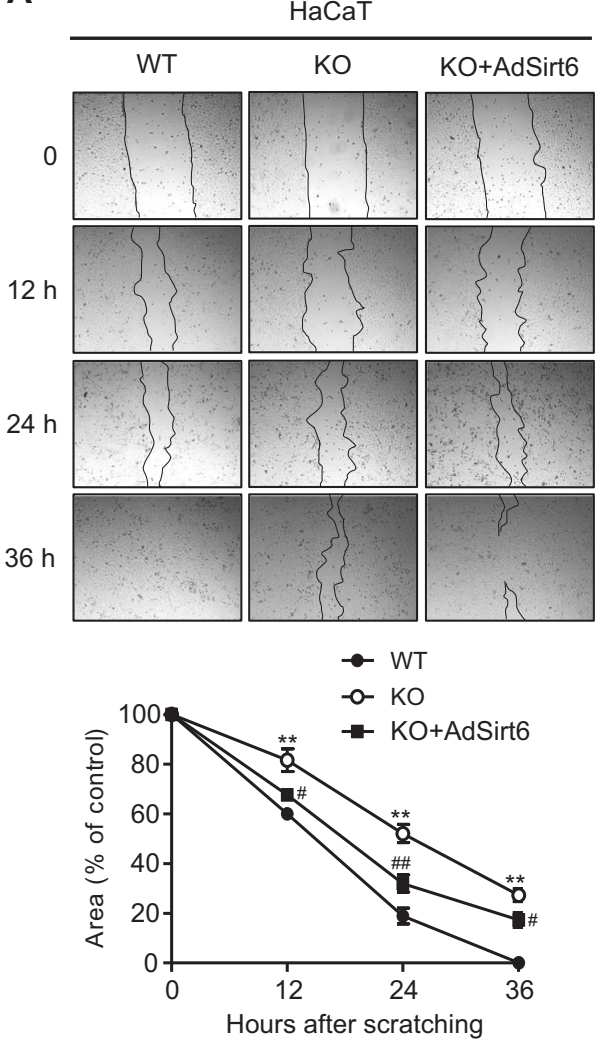

B
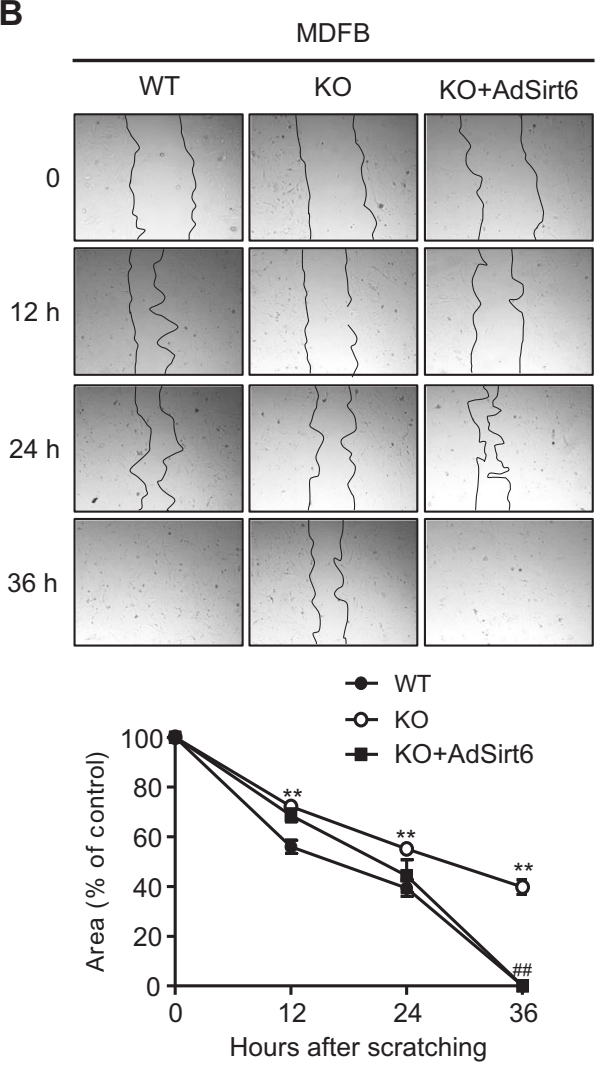

Fig. 6 Delayed migration of skin keratinocytes and fibroblasts in wound scratch assays. Bone marrow macrophages $\left(B M M s, 2 \times 10^{6}\right)$ isolated from WT or KO mice were treated with TNF-a $(10 \mathrm{ng} / \mathrm{mll}), \mathrm{LL}-1 \beta(10 \mathrm{ng} / \mathrm{mll})$, and IL-6 $(10 \mathrm{ng} / \mathrm{ml})$ for $3 \mathrm{~h}$, and then conditioned medium (CM) was harvested. Scratch wounds were made in monolayers of HaCaT (a) and MDFB cells (b). For rescue experiments, KO BMMs were transduced with AdSirt6. Representative images show the progression of wound closure at the indicated times. The scratch area is indicated by a dotted line. The rate of wound closure was calculated by measuring the scratch area under the same magnification at different times. Values represent the mean \pm SEM $(n=8){ }^{* *} p<0.01$ vs. WT; ${ }^{\#} p<0.05$; and ${ }^{\# \#} p<0.01$ vs. KO 


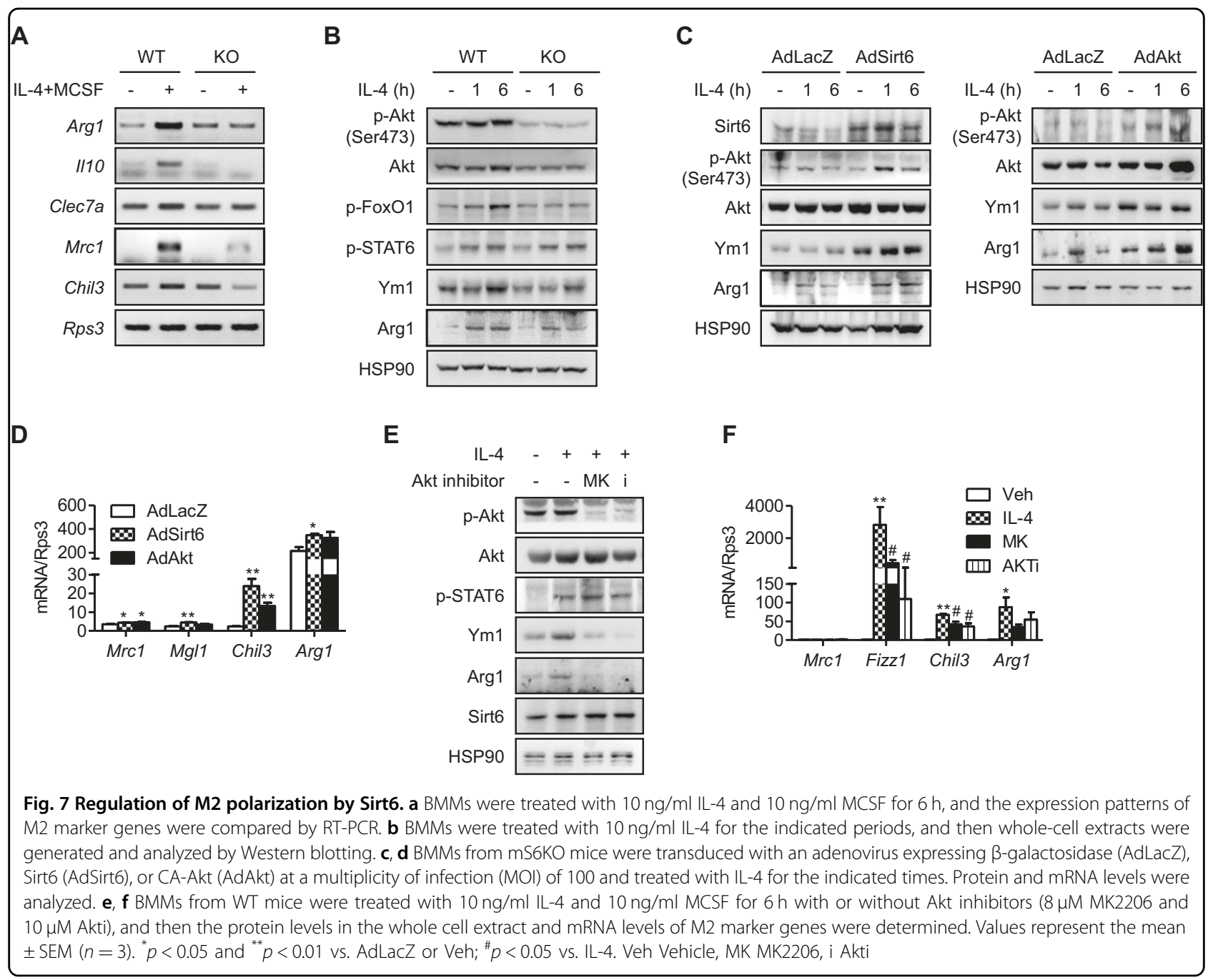

similar in BMMs from $\mathrm{mS6KO}$ and WT mice (Fig. 7b), indicating that the change in M2 marker expression was mediated through the downregulation of components of the PI3K-Akt pathway and not through the JAK2-STAT6 pathway. To further investigate the regulation of the PI3K-Akt signaling pathway by Sirt6 under M2-polarizing conditions, we transduced BMMs with either Sirt6 (AdSirt6) or a constitutively active form of Akt (AdAkt). Treatment with IL-4 led to a moderate increase in M2 marker protein expression in the BMMs from $\mathrm{mS} 6 \mathrm{KO}$ mice expressing Sirt6 or Akt (Figs. 7c and S3B). Accordingly, the levels of M2 marker genes were increased in the Sirt6- or Akt-overexpressing KO cells (Fig. 7d). The role of Akt signaling in the enhancement of M2 polarization was further tested by blocking Akt signals with MK2206 or Akti. When WT BMMs were pretreated with these Akt inhibitors prior to stimulation with IL-4, Akt phosphorylation and M2 marker gene expression were markedly suppressed (Figs. 7e, S3C, and 7F). Finally, consistent with these in vitro results, in vivo results showed that Akt phosphorylation was increased in skin wounds in WT mice but was significantly impaired in skin wounds in $\mathrm{KO}$ mice (Fig. S4).

\section{Discussion}

In this study, we demonstrated that myeloid cell-specific Sirt6 deficiency leads to delayed wound closure compared to WT control. This aberrant wound closure pattern was associated with the augmented infiltration of macrophages that failed to phenotypically switch from M1 to M2 macrophages.

Previously, Thandavarayan et al. ${ }^{26}$ observed that Sirt6 knockdown impairs diabetic wound closure with concomitantly increased levels of oxidative stress, inflammatory cytokines, and NF- $\mathrm{KB}$ activation in skin wounds. Similarly, the sirtuin activator resveratrol accelerates wound repair by increasing keratinocyte proliferation, while the sirtuin inhibitor sirtinol retards wound 
closure $^{27}$. More recently, Hu et al. ${ }^{28}$ showed that Sirt6 KO mice display delayed and incomplete healing of the cornea after wounding. These reports suggest a beneficial role for sirtuin (specifically Sirt6) activation in wound healing. Based on this background, we aimed to understand how a myeloid cell-specific Sirt6 deficiency would affect macrophage infiltration and wound closure using $\mathrm{mS6KO}$ mice. Our results showed that the number of macrophages in excisional wounds was markedly increased in $\mathrm{mS6KO}$ mice. The significantly increased expression of a macrophage chemoattractant, CCL2, in $\mathrm{mS6KO}$ mice is possibly responsible for the increased infiltration of macrophages seen in the wounds of this strain compared to those of WT mice. Remarkably, we clearly demonstrated heterogeneity in the macrophage populations recruited to the wounds of $\mathrm{mS6KO}$ mice. The expression levels of established M1-specific marker genes were upregulated in skin wounds in $\mathrm{mS6KO}$ mice, while the levels of M2 marker genes were downregulated. These results imply that the wound environment in $\mathrm{mS6KO}$ mice favors the proinflammatory M1 status.

Ample evidence points to a phenotypic switch from an M1 to an M2 macrophage in the process of wound repair $^{29}$. In the early inflammatory phase, M1 macrophages are the predominant cells in the tissue and are involved in the clearance of pathogens and dead cells and in the modulation of the adaptive immune system. In the later proliferative phase, M2 macrophages contribute to the resolution of inflammation and tissue remodeling. While STAT6 has been shown to be required for IL-4 to exert its effects ${ }^{24}$, the level of p-STAT6 was shown to be unaffected by Sirt6 deficiency, indicating that IL-4 impacts other signaling pathways. We observed enhanced levels of p-Akt in BMMs after exposure to IL-4, a finding consistent with that of a previous study showing that the activation of the PI3K-Akt pathway results in stronger polarization of macrophages toward the M2 phenotype $^{25}$. Moreover, the transduction of KO BMMs with CA-Akt selectively restored the expression of M2 marker genes. These results indicate that the activation of the PI3K-Akt pathway is required for the expression of M2 markers; however, future studies are needed to determine how Sirt6 deficiency affects the PI3K-Akt pathway.

Using in vivo and in vitro approaches, we demonstrated that myeloid cell-specific Sirt6 deficiency utilizes the following mechanisms to delay wound closure. First, more proinflammatory cytokines such as TNF- $\alpha$, IL- $1 \beta$, and IL6 were produced in $\mathrm{mS6KO}$ mice. It is well known that M1-type macrophages are a source of proinflammatory cytokines. These cytokines alter the normal functioning of epithelial cells and dermal fibroblasts and ultimately delay the rates of re-epithelialization (epithelium closure) and wound closure (dermis closure ${ }^{29}$. Second, we observed a lower number of vWF-positive cells in mS6KO mice than in WT mice. M1 macrophages are described as having an antiangiogenic profile, which is considered detrimental to recovery after an inflammatory event ${ }^{30,31}$. Third, the delayed wound closure observed in $\mathrm{mS6KO}$ mice was accompanied by decreases in the migration of keratinocytes and dermal fibroblasts, suggesting that myeloid cellspecific Sirt6 deficiency might impair epithelial and dermal closure by suppressing cell migration. Although the underlying mechanisms by which myeloid cell-specific Sirt6 deficiency suppresses keratinocyte and dermal fibroblast migration remain elusive, these findings suggest, for the first time, that myeloid cell-specific Sirt6 deficiency has a deleterious role in excisional wound healing. Therefore, myeloid cell-specific Sirt6 activation might be a therapeutic strategy for accelerating wound healing.

\section{Acknowledgements}

This research was supported by grants from the Medical Research Center Program (2017R1A5A2015061) and Basic Science Research Program (2017R1A2B2005730, 2018R1D1A1B07050772, and 2019R1A2C2002281) through the National Research Foundation of Korea (NRF), which is funded by the Korean government, and by a fund of the Biomedical Research Institute, Chonbuk National University Hospital (to S.K.Y.).

\section{Author details}

'Department of Biochemistry and Molecular Biology, Chonbuk National University Medical School, Jeonju, Jeonbuk 54896, Republic of Korea. ${ }^{2}$ College of Pharmacy, Woosuk University, Wanju, Jeonbuk 55338, Republic of Korea. ${ }^{3}$ Department of Dermatology and Research Institute of Clinical Medicine, Chonbuk National University Medical School, Jeonju, Jeonbuk 54896, Republic of Korea. ${ }^{4}$ Biomedical Research Institute, Chonbuk National University Hospital, Jeonju, Jeonbuk 54907, Republic of Korea

Conflict of interest

The authors declare that they have no conflict of interest.

\section{Publisher's note}

Springer Nature remains neutral with regard to jurisdictional claims in published maps and institutional affiliations.

Supplementary information accompanies this paper at https://doi.org/ 10.1038/s12276-019-0248-9.

Received: 25 July 2018 Revised: 27 December 2018 Accepted: 16 January 2019.

Published online: 26 April 2019

\section{References}

1. Eming, S. A., Krieg, T. \& Davidson, J. M. Inflammation in wound repair: molecular and cellular mechanisms. J. Invest. Dermatol. 127, 514-525 (2007).

2. Kanno, E. et al. Wound healing in skin promoted by inoculation with Pseudomonas aeruginosa PAO1: The critical role of tumor necrosis factor-a secreted from infiltrating neutrophils. Wound Repair Regen. 19, 608-621 (2011).

3. Kim, M. H. et al. Dynamics of neutrophil infiltration during cutaneous wound healing and infection using fluorescence imaging. J. Invest. Dermatol. 128, 1812-1820 (2008)

4. Al-Mulla, F., Leibovich, S. J., Francis, I. M. \& Bitar, M. S. Impaired TGF- $\beta$ signaling and a defect in resolution of inflammation contribute to delayed wound healing in a female rat model of type 2 diabetes. Mol. Biosyst. 7, 3006-3020 (2011). 
5. Numata, Y. et al. The accelerating effect of histamine on the cutaneous wound-healing process through the action of basic fibroblast growth factor. J. Invest. Dermatol. 126, 1403-1409 (2006).

6. Plasari, G. et al. Nuclear factor I-C links platelet-derived growth factor and transforming growth factor $\beta 1$ signaling to skin wound healing progression. Mol. Cell Biol. 29, 6006-6017 (2009).

7. Barrientos, S., Stojadinovic, O., Golinko, M. S., Brem, H. \& Tomic-Canic, M. Growth factors and cytokines in wound healing. Wound Repair Regen. 16, 585-601 (2008).

8. Goren, l. et al. A transgenic mouse model of inducible macrophage depletion: effects of diphtheria toxin-driven lysozyme M-specific cell lineage ablation on wound inflammatory, angiogenic, and contractive processes. Am. J. Pathol. 175, 132-147 (2009)

9. Mirza, R., DiPietro, L. A. \& Koh, T. J. Selective and specific macrophage ablation is detrimental to wound healing in mice. Am. J. Pathol. 175, 2454-2462 (2009).

10. Jablonski, K. A. et al. Novel markers to delineate murine M1 and M2 macrophages. PLOS ONE 10, e0145342 (2015).

11. Lucas, T. et al. Differential roles of macrophages in diverse phases of skin repair. J. Immunol. 184, 3964-3977 (2010).

12. Kugel, S. \& Mostoslavsky, R. Chromatin and beyond: the multitasking roles for SIRT6. Trends Biochem. Sci. 39, 72-81 (2014).

13. Kawahara, T. L. et al. SIRT6 links histone H3 lysine 9 deacetylation to NF-KBdependent gene expression and organismal life span. Cell 136, 62-74 (2009).

14. Michishita, E. et al. Cell cycle-dependent deacetylation of telomeric histone $\mathrm{H3}$ lysine K56 by human SIRT6. Cell Cycle 8, 2664-2666 (2009).

15. Song, M. Y., Wang, J., Ka, S. O., Bae, E. J. \& Park, B. H. Insulin secretion impairment in Sirt6 knockout pancreatic $\beta$ cells is mediated by suppression of the FoxO1-Pdx1-Glut2 pathway. Sci. Rep. 6, 30321 (2016).

16. Woo, S. J. et al. Myeloid sirtuin 6 deficiency accelerates experimental rheumatoid arthritis by enhancing macrophage activation and infiltration into synovium. EBioMedicine 38, 228-237 (2018).

17. Kaidi, A., Weinert, B. T., Choudhary, C. \& Jackson, S. P. Human SIRT6 promotes DNA end resection through CtIP deacetylation. Science 329, 1348-1353 (2010).

18. Dominy, J. E. Jr. et al. The deacetylase Sirt6 activates the acetyltransferase GCN5 and suppresses hepatic gluconeogenesis. Mol. Cell 48, 900-913 (2012).
19. Bhardwaj, A., Bhardwaj, A. \& Das, S. SIRT6 deacetylates PKM2 to suppress its nuclear localization and oncogenic functions. Proc. Natl Acad. Sci. USA 113 E538-E547 (2016)

20. Jang, H. Y., Gu, S., Lee, S. M. \& Park, B. H. Overexpression of sirtuin 6 suppresses allergic airway inflammation through deacetylation of GATA3. J. Allergy Clin. Immunol. 138, 1452-1455 (2016).

21. Lee, Y. et al. Myeloid sirtuin 6 deficiency causes insulin resistance in high-fat diet-fed mice by eliciting macrophage polarization toward an M1 phenotype. Diabetes 66, 2659-2668 (2017).

22. Lee, H. S. et al. Overexpression of sirtuin 6 suppresses inflammatory responses and bone destruction in mice with collagen-induced arthritis. Arthritis Rheum 65, 1776-1785 (2013).

23. $\mathrm{Ko}, \mathrm{H}$. R. et al. Akt1-Inhibitor of DNA binding2 is essential for growth cone formation and axon growth and promotes central nervous system axon regeneration. Elife 5, e20799 (2016).

24. Barrett, J. P., Minogue, A. M., Falvey, A. \& Lynch, M. A. Involvement of IGF-1 and Akt in $\mathrm{M} 1 / \mathrm{M} 2$ activation state in bone marrow-derived macrophages. Exp. Cell Res. 335, 258-268 (2015)

25. Gordon, S. \& Martinez, F. O. Alternative activation of macrophages: mechanism and functions. Immunity 32, 593-604 (2010).

26. Thandavarayan, R. A. et al. Sirtuin-6 deficiency exacerbates diabetes-induced impairment of wound healing. Exp. Dermatol 24, 773-778 (2015).

27. Spallotta, F. et al. A nitric oxide-dependent cross-talk between class I and II histone deacetylases accelerates skin repair. J. Biol. Chem. 288, 11004-11012 (2013).

28. $\mathrm{Hu}, \mathrm{X}$. et al. Sirt6 deficiency impairs corneal epithelial wound healing. Aging $\mathbf{1 0}$ 1932-1946 (2018)

29. Mahdavian Delavary, B., van der Veer, W. M., van Egmond, M., Niessen, F. B. \& Beelen, R. H. Macrophages in skin injury and repair. Immunobiology 216 753-762 (2011).

30. Chambers, S. E., O'Neill, C. L., O'Doherty, T. M., Medina, R. J. \& Stitt, A. W. The role of immune-related myeloid cells in angiogenesis. Immunobiology $\mathbf{2 1 8}$ 1370-1375 (2013).

31. Mosser, D. M. \& Edwards, J. P. Exploring the full spectrum of macrophage activation. Nat. Rev. Immunol. 8, 958-969 (2008). 